\title{
TRANSLATION INVARIANTS FOR PERIODIC DENJOY-CARLEMAN CLASSES
}

\author{
P. X. GALLAGHER
}

(Communicated by Andrew M. Bruckner)

\begin{abstract}
The Denjoy-Carleman classes in real $C^{\infty}(\mathbb{R} / \mathbb{Z})$ on which the derivative sequence $f^{(n)}(x)$ at any point is a complete set of invariants are exactly the ones on which the integrals of products of derivatives $f^{\left(n_{1}\right)} \ldots f^{\left(n_{r}\right)}$ are a complete set of invariants up to translation.
\end{abstract}

The object of this note is to combine the Adler-Konheim complete set of invariants on the space of real integrable functions on a locally compact abelian group, up to translation, with the Denjoy-Carleman theorem on "quasi-analytic" classes of $C^{\infty}(\mathbb{R} / \mathbb{Z})$ to give the result (Theorem 3 ) in the abstract.

Theorem 1 (Adler-Konheim [1]). Let $G$ be a locally compact abelian group. The functions $\varphi_{r}$ in $L^{1}\left(G^{r}\right)$ for $r=1,2,3, \ldots$ defined for $f$ in real $L^{1}(G)$ by

$$
\varphi_{r}(\mathbf{y})=\int_{G} f\left(x+y_{1}\right) \cdots f\left(x+y_{r}\right) d x
$$

are a complete set of invariants up to translation. If the Fourier transform of $f$ has no zeros, then $f$ is determined up to translation by $\varphi_{1}, \varphi_{2}, \varphi_{3}$.

For $f$ in $C^{\infty}\left(\mathbb{R} / \mathbb{Z}\right.$ ) we define functions $f_{r}$ on $\mathbb{N}^{r}$ (with $\mathbb{N}=\{0,1,2, \ldots\}$ ) for $r=1,2,3, \ldots$ by

$$
f_{r}(\mathbf{n})=\int_{0}^{1} f^{\left(n_{1}\right)}(x) \cdots f^{\left(n_{r}\right)}(x) d x .
$$

These functions are not a complete set of invariants for $C^{\infty}(\mathbb{R} / \mathbb{Z})$ up to translation since, e.g., $g(x)+g\left(x+\frac{1}{4}\right)$ and $g(x)+g\left(x+\frac{1}{2}\right)$, for any function $g$ in $C^{\infty}(\mathbb{R} / \mathbb{Z})$ supported $(\bmod 1)$ on an interval of length $\frac{1}{4}$ but $\not \equiv 0$, will have the same $f_{r}$ 's but will not be translates.

If $f$ is entire with period 1 , then $f_{r}$ determines $\varphi_{r}$, since integrating products of Taylor series gives

$$
\varphi_{r}(\mathbf{y})=\sum_{\mathbf{n}} f_{r}(\mathbf{n}) \frac{\mathbf{y}^{\mathbf{n}}}{\mathbf{n} !}
$$

Received by the editors April 15, 1992.

1991 Mathematics Subject Classification. Primary 26E10.

Key words and phrases. Quasi-analytic functions, Denjoy-Carleman classes, translation invariants for periodic functions. 
with $\mathbf{y}^{\mathbf{n}}=y_{1}^{n_{1}} \cdots y_{r}^{n_{r}}$ and $\mathbf{n} !=n_{1} ! \cdots n_{r} !$. If $f$ is only real-analytic on $\mathbb{R} / \mathbb{Z}$, i.e., analytic with period 1 in a strip neighborhood of $\mathbb{R}$, then $f_{r}$ still determines $\varphi_{r}$. This may be shown by integrating products of iterated Taylor series

$$
f(x+y)=\sum_{\nu_{1}} \cdots \sum_{\nu_{s}} f^{(n)}(x) \frac{\eta_{1}^{\nu_{1}} \cdots \eta_{s}^{\nu_{s}}}{\nu_{1} ! \cdots \nu_{s} !}
$$

with $n=\nu_{1}+\cdots+\nu_{s}$ and $y=\eta_{1}+\cdots+\eta_{s}$ with small $\eta_{i}$. We omit the details since the result is contained in Theorem 3 as the case $m_{n}=n$ ! .

For a positive sequence $\mathbf{m}=\left(m_{0}, m_{1}, \ldots\right)$ and a real interval $I$, the DenjoyCarleman class $C_{\mathrm{m}}(I)$ consists of the functions $f$ in $C^{\infty}(I)$ for which there are positive constants $a, b$ depending on $f$ such that

$$
\sup \left|f^{(n)}\right| \leq a b^{n} m_{n} \quad(n \in \mathbb{N})
$$

or, equivalently,

$$
\left|f_{r}(\mathbf{n})\right| \leq a^{r} b^{|\mathbf{n}|} m_{\mathbf{n}} \quad\left(\mathbf{n} \in \mathbb{N}^{r} ; r=1,2,3, \ldots\right)
$$

with $|\mathbf{n}|=n_{1}+\cdots+n_{r}$ and $m_{\mathbf{n}}=m_{n_{1}} \cdots m_{n_{r}}$. From $\left(1_{\mathrm{D}}\right)$ to $\left(1_{\mathrm{I}}\right)$ is immediate. The opposite direction follows from

$$
\sup \left|f^{(n)}\right|=\lim \left\|f^{(n)}\right\|_{r} \quad(r \rightarrow \infty)
$$

and

$$
\left\|f^{(n)}\right\|_{r}^{r}=f_{r}(n, \ldots, n) \quad(r \text { even }) .
$$

Theorem 2 (Denjoy-Carleman $[2,3,5])$. The derivative sequence $f^{(n)}$ at any point in $I$ is a complete set of invariants for $C_{\mathbf{m}}(I)$ if and only if

$$
\sum_{n=0}^{\infty}\left(\inf _{k \geq n} m_{k}^{1 / k}\right)^{-1}=\infty \text {. }
$$

The same holds with I replaced by $\mathbb{R} / \mathbb{Z}, C_{\mathbf{m}}(\mathbb{R} / \mathbb{Z})$ being defined similarly.

Theorem 3. The integrals $f_{r}(\mathbf{n})$ for $\mathbf{n}$ in $\mathbb{N}^{r}(r=1,2,3, \ldots)$ are a complete set of invariants on real $C_{\mathrm{m}}(\mathbb{R} / \mathbb{Z})$ up to translation if and only if (2) holds. If (2) holds and $f$ in real $C_{\mathbf{m}}(\mathbb{R} / \mathbb{Z})$ has no vanishing Fourier coefficient, then the $f_{r}$ with $r=1,2,3$, which amounts to

$$
\int_{0}^{1} f, \int_{0}^{1}\left(f^{(n)}\right)^{2}, \int_{0}^{1}\left(f^{(n)}\right)^{2} f^{(k)} \text { for } n, k \text { in } \mathbb{N}, k \leq n,
$$

suffice to determine $f$ up to translation.

For $n$ a positive integer and $\mathbf{m}=\left(m_{0}, m_{1}, \ldots\right)$, put $\mathbf{m}^{(n)}=\left(m_{n}, m_{n+1}, \ldots\right)$. From $\left(1_{\mathrm{D}}\right)$ it follows that $f \rightarrow f^{(n)}$ maps $C_{\mathbf{m}}(I)$ onto $C_{\mathbf{m}^{(n)}}(I)$, with $a, b$ replaced by $a b^{n}, b$. Condition (2) is preserved by $\mathbf{m} \rightarrow \mathbf{m}^{(n)}$ : For this we make use of the fact, due to Carleman [2, p. 105] and used by him for the same purpose, that for series with positive terms,

$$
\sum_{n=2}^{\infty} c_{n}^{1-1 / n} \leq \sum_{n=2}^{\infty} c_{n}+2\left(\sum_{n=2}^{\infty} c_{n}\right)^{1 / 2} .
$$

For the convenience of the reader we repeat Carleman's proof. For each $\lambda>1$, the terms with $c_{n}^{-1 / n} \leq \lambda$ contribute $\leq \lambda s$ to the series on the left, $s$ being 
the series on the right. For the remaining terms, $c_{n}^{1-1 / n} \leq \lambda^{-(n-1)}$, giving a contribution $\leq 1 /(\lambda-1)$. Choosing $\lambda=1+s^{-1 / 2}$ gives the total bound $s+2 s^{1 / 2}$. For the preservation of (2), it suffices to show that

$$
\sum_{n}\left(\inf _{k \geq n} m_{k}^{1 / k}\right)^{-1} \text { converges if } \sum_{n}\left(\inf _{k \geq n} m_{k+1}^{1 / k}\right)^{-1} \text { converges. }
$$

For this we may assume all $m_{n} \geq 1$. This implies

$$
\left(\inf _{k \geq n+1} m_{k}^{1 / k}\right)^{-1} \leq\left(\left(\inf _{k \geq n} m_{k+1}^{1 / k}\right)^{-1}\right)^{1-1 / n}
$$

which with (3) gives (4).

Proof of Theorem 3. Let $\mathbf{m}$ satisfy (2). For $f$ in $C_{\mathbf{m}}(\mathbb{R} / \mathbb{Z})$, each $D^{\mathbf{n}} \varphi_{r}$ with $D^{\mathbf{n}}=D^{n_{1}} \cdots D^{n_{r}}$ belongs to $C_{\mathbf{m}}\left(n_{j}\right)$ in the $j$ th variable, the other variables being held fixed, with $a, b$ replaced by $a^{r} b^{|\mathbf{n}|} m_{\mathbf{n}} / m_{n_{j}}, b$. This fact and Theorem 2 show that $f_{r}$, i.e.,

$$
f_{r}(\mathbf{n})=D^{(\mathbf{n})} \varphi_{r}(0, \ldots, 0) \text { for all } \mathbf{n}
$$

determines

$$
D^{(\mathbf{n})} \varphi_{r}\left(y_{1}, 0, \ldots, 0\right) \text { for all } \mathbf{n}, y_{1} .
$$

Taking $r$ steps in this way leads to

$$
D^{(\mathbf{n})} \varphi_{r}\left(y_{1}, \ldots, y_{r}\right) \text { for all } \mathbf{n}, \mathbf{y}
$$

and, in particular, to $\varphi_{r}$. The first statement in Theorem 1 now shows that the $f_{r}$ determine $f$ up to translation. The relation

$$
f_{r}\left(n_{1}+1, n_{2}, \ldots, n_{r}\right)+\cdots+f_{r}\left(n_{1}, \ldots, n_{r-1}, n_{r}+1\right)=0 \quad\left(\mathbf{n} \in \mathbb{N}^{r}\right)
$$

shows that for $r=1,2,3$ the $f_{r}$ are determined by

$$
f_{1}(0), f_{2}(n, n), f_{3}(n, n, k) \text { for } n, k \in \mathbb{N}, k \leq n \text {. }
$$

If $\mathbf{m}$ fails (2), then, as Mandelbrojt has shown [4, 5], there are real $g$ in $C_{\mathbf{m}}(\mathbb{R} / \mathbb{Z})$ with arbitrarily small support $(\bmod 1)$ but $\not \equiv 0$. The functions $g(x)+g\left(x+\frac{1}{4}\right)$ and $g(x)+g\left(x+\frac{1}{2}\right)$, for such $g$ supported in an interval of length $\frac{1}{4}$, belong to $C_{\mathrm{m}}(\mathbb{R} / \mathbb{Z})$ and, as before, have the same $f_{r}$ 's but are not translates.

\section{REFERENCES}

1. R. L. Adler and A. G. Konheim, A note on translation invariants, Proc. Amer. Math. Soc. 13 (1962), 425-428.

2. T. Carleman, Les fonctions quasi analytiques, Gauthier-Villars, Paris, 1926.

3. S. Mandelbrojt, Series de Fourier et classes quasi-analytiques de fonctions, Gauthier-Villars, Paris, 1935.

4. __ Infinitely differentiable functions, Duke Math. J. 11 (1944), 341-349.

5. __ Series adherentes regularisation des suites applications, Gauthier-Villars, Paris, 1952. 